\title{
BlindMobi: A system for bus identification, based on Bluetooth Low Energy, for people with visual impairment
}

\author{
Hilson G. V. de Andrade ${ }^{1}$, David de M. Borges ${ }^{1}$, Leandro H. C. Bernardes ${ }^{1}$, \\ João Lucas A. de Albuquerque ${ }^{1}$, Abel G. da Silva-Filho ${ }^{2}$
}

${ }^{1}$ Federal Institute of Education, Science and Technology of Pernambuco (IFPE) Av. Prof. Luís Freire, 500 - Cid. Universitária - 50.740-540 - Recife - PE - Brazil

${ }^{2}$ Informatics Center - Federal University of Pernambuco (UFPE)

Av. Jorn. Aníbal Fernandes, s/n - Cid. Universitária - 50.740-560 - Recife - PE - Brazil

\begin{abstract}
This paper presents a bus detection system based on Bluetooth low energy $(B L E)$ technology that aims to ease the traveling of blind people, in large urban centers. The proposed system consists of two subsystems: one embedded hardware on the buses and the other running on the user mobile device. From the device embedded on the bus, BLE beacons containing itinerary information and bus acceleration are sent and read by the application running on the mobile device. Through this information and using the machine learning algorithm, the application is able to identify the approach and arrival of the bus, guiding the user. A complete system prototype has been constructed and tested to validated the proposed system, where a rating rate of $91.5 \%$ was obtained, indicating the feasibility of the proposal.
\end{abstract}

\section{Introduction}

Although present in the Universal Declaration of Human Rights [United Nation 1948], the right to free movement is not guaranteed to everyone, especially people with visual impairment. This public, which globally amounts to about 285 million people [Pascolini and Mariotti 2012], finds a great barrier to autonomous locomotion, with the use of collective transportation, especially in large urban centers of developing countries like Brazil. Given this scenario, the development of assistive technologies aimed at helping this public to use public transport is crucial. In addition to considering the constraints imposed by user conditions, such technology should be simple enough to integrate existing infrastructure with a low deployment and maintenance cost, minimizing the need for additional infrastructure for its operation, such as adjustments at boarding points, increasing the capacity of wireless or cellular networks, as well as adjustments in collective vehicles.

In this context, this article presents the BlindMobi proposal, a low cost system, without the need of additional infrastructure to the urban environment, which allows the user to identify approach and stop at bus stops (or subway stations), thus contributing to guarantee the right to free movement of visually impaired public transport users. In Session 2, two related works are presented, whose objectives are similar to the proposal of the article, however presenting differences in implementation, regarding communication technology and support infrastructure. Such differences are detailed in Section 3, which presents a description of the communication technology used, as well as detailed hardware and software components of the proposal. In Section 4, we present the constructive details 
of the prototype used for validation of the proposal and the results obtained in field tests. Finally, Section 5 presents the conclusions and Future Work.

\section{Related Work}

In view of the need for methods to assist the use of collective transportation for the partially or totally blind, previous research proposes systems capable of identifying the approach of the buses of the boarding stations and issuing sound alerts, thus enabling the use of these buses by all people.

Based on a previous study that presented the requirements for the interconnection of assistive technology devices [Gill 2003], it was proposed a Bus Notification System [Lim et al. 2008] using a communication through bluetooth technology between the bus, the bus-stop and the users mobile device (bluetooth client). To validate the proposal, a prototype was developed where a server was placed at the Bus-stop, connected through a bluetooth adapter with the user's device and a dedicated card to connect with the bus, thus receiving the signal of all transport passing through the place of boarding, see Figure 1. In addition to predicting the need to establish connections between the users' devices with the server, and the server with the buses, which imposes limitations on the system, this proposal demands the implementation of additional infrastructure in the places of boarding, for accommodation of the server itself .

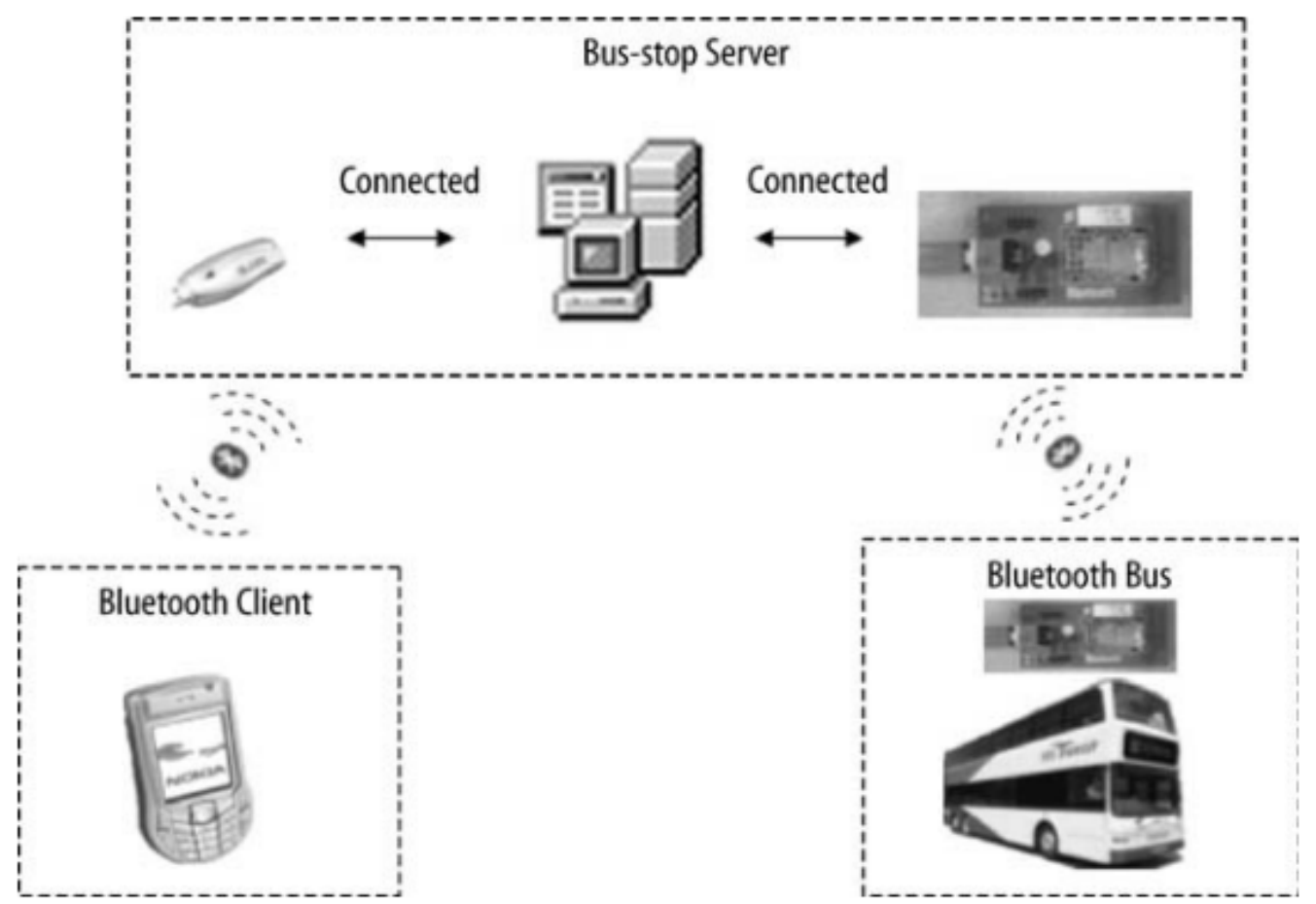

Figure 1. A Bus Notification System based on bluetooth [Lim et al. 2008]

Also within the concept of the use of short-range wireless communication for the notification of bus approach users, we have another approach based on the use of RFID [Kalbani et al. 2015]. In this research, two subsystems (one in the bus and the other in the station) were proposed, connected to a database, where the user data is inserted at the moment of the ticket purchase. Both the bus station, the bus and the passengers have an 
RFID tag and it is used in the communication between the station and the bus, when the two change their identifiers and verify if the bus will pass in the bus stop in question, as well as if there are users with special needs, so that the bus is announced by the speaker, see Figure 2.

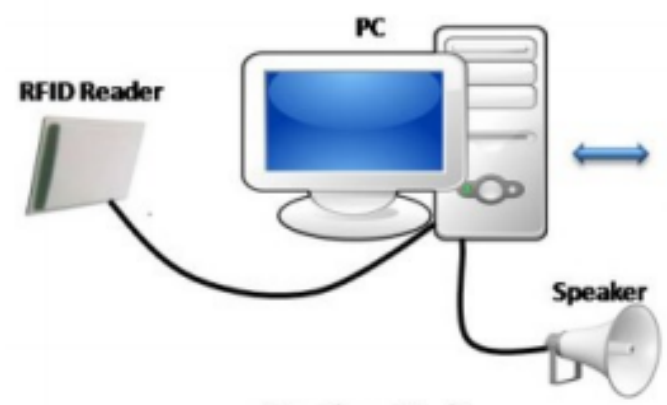

Station Unit

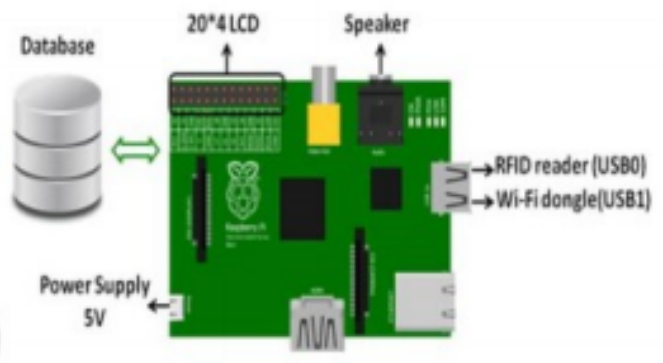

Bus Unit

Figure 2. RFID-based system diagram [Kalbani et al. 2015]

In both systems described above, in addition to the need to deploy additional infrastructure at the boarding stations, which represents an increase in the cost of deploying and maintaining the solution, there is also a need for a connection to a central server or database, to periodically update the position of the same, in relation to the user. In the system proposed in this work, the need for additional infrastructure in the places of boarding is replaced by the use of an application to be deployed in the user's smartphone, whose interaction with the device to be deployed in the bus (described in Section 3.2) will occur from of the reading of periodic messages (beacons), that is, without the need of establishing connection between the devices involved. In addition to the information of the itinerary, to be used by the application of the system for the generation of audible messages, such beacons will also be used to determine the distance of the same with respect to the user, based on the calculation performed according to RSSI (Received Signal Strength Indicator ) [Alawi 2011] and vehicle acceleration, as detailed in section 4.

\section{Proposed System}

The basic architecture of the BlindMobi System, whose main objective is to allow the user, from voice messages, the identification of the approach, the itinerary and the stop at the place of boarding by bus or any other terrestrial collective means of transportation, is composed by an electronic device to be embedded in the vehicle (BlindMobi Box) and by an application to be installed in the user's smartphone (BlindMobi App). As described in the previous section, the exchange of information between the BlindMobi Box module and the BlindMobi App software is done through the wireless communication technology BLE (detailed in Section 3.1), without the connection establishment, that is, the application reads the periodic messages sent and extracts the information necessary for its operation.

A functional decomposition of the BlindMobi system is shown in Figure 3. The areas marked in red delimit the hardware to be embedded in the vehicle (BlindMobi Box), showing the interfaces of the same with the power system, from where the power source 
of the system will be obtained, and with the control panel of the route display of the bus from which the route information to be announced for the application can be obtained. On the right is the functional decomposition of the developed application (BlindMobi App), which will be supported by the mobile operating system of the user's smartphone and whose functionalities are described in Section 3.3. The system proposed search meets the following requirements:

- The system should be generic enough to be applied in different countries.

- The system should generate a clear guidance message about the buses (or others) arriving at the station.

- The system should be useful for non-blind people (besides the blind people).

- The system will use unlicensed radio frequency bands reserved internationally for ISM applications including bluetooth.

- The system will have no restriction on the number of concurrent users.

- The system will produce an accurate and clear voice message within frequency range $250 \mathrm{~Hz}-8 \mathrm{kHz}$.
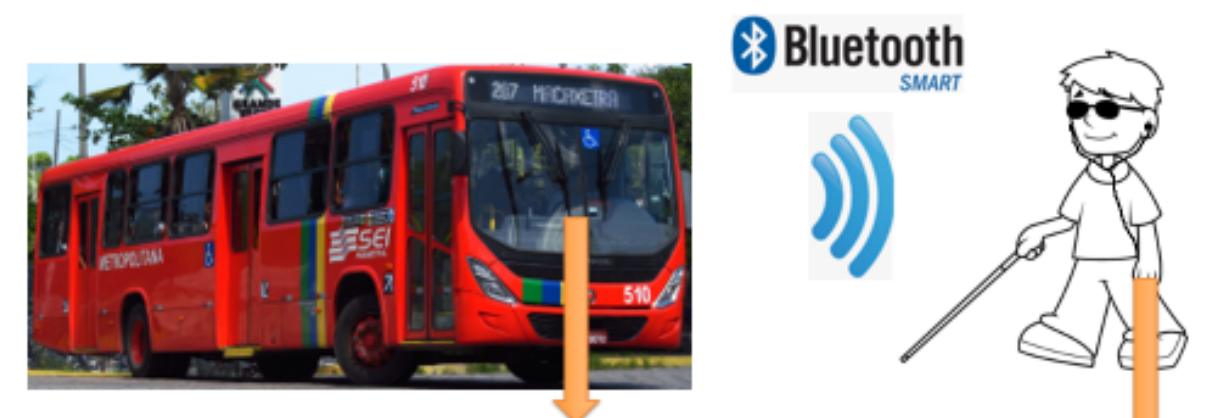

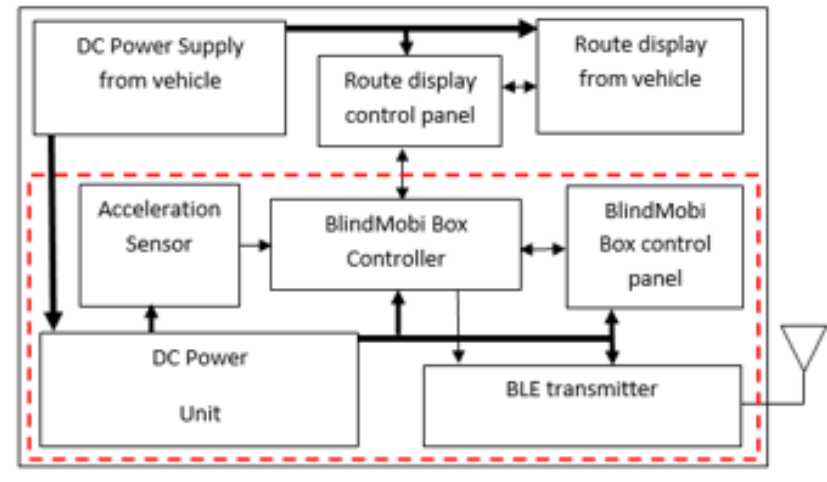

BlindMobi Box

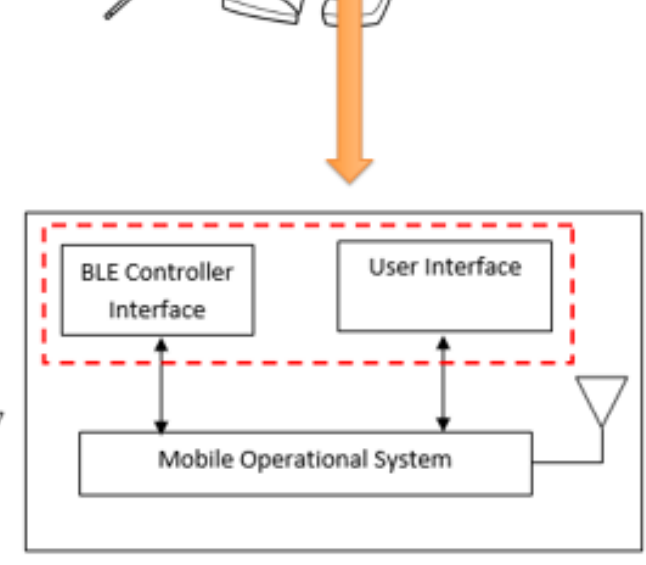

BlindMobi App

Figure 3. Functional Decomposition of the System BlindMobi

\subsection{Bluetooth Low Energy Technology}

Among the various low-power wireless technologies such as RFID, ZigBee and 6LoPan, Bluetooth Low Energy (BLE) stands out for its energy efficiency and ease of integration with mobile devices. Successor of classic bluetooth and also known as bluetooth smart or bluetooth 4.0, BLE unies the advantages of unmanned power constrained IoT applications and Bluetooth-enabled smart devices, it is increasingly adopted, and BLE beacons one of its most promising applications. The ease of integration between off-the-shelf BLE beacons and smartphones in particular, has promoted diverse IoT use cases, especially 
among the emerging unmanned IoT applications, requiring less human efforts to do any task [Atzori et al. 2010], fitting perfectly into applications aimed at assistive technologies, as proposed in this work.

According to the survey recently presented [Jeon et al. 2018], BLE beacons have been employed in a wide range of IoT innovations, for example, improving shopper's experience, museum guiding, indoor localization and tracking, helping the blind or disabled, energy saving smart ofces, managing smart homes and warehouses, locating BLE devices with beacons using fingerprinting and so on. In the prototype used for validation of the BlindMobi System - as described in Section 4, BLE was used not only to send beacons containing the itinerary and speed of the bus, but also to determine the distance from the bus to the user, fundamental information for the operation of the system. For such, it was used two bluetooth fingerprinting-based algorithms for localization [Zhang et al. 2013] [Bertuletti et al. 2016], obtaining satisfactory results, as presented in section 4.3.

In this way, the choice of BLE technology for the BlindMobi System met the market's and engineering requirements of the proposal, optimizing the energy consumption of the devices and avoiding the use of dedicated devices such as GPS modules. In the prototype used for system validation, the native BLE transmitter of SoC NRF52832, manufactured by Nordic, was used as detailed in section 4.1.

\subsection{BlindMobi Box description}

The main function of this subsystem, to be deployed in the vehicle, is the generation and sending of the BLE beacons, which will be read by the application installed on the user's mobile device.

As shown in Figure 4, the process starts with the definition of the vehicle's route, which can be entered directly by the driver through a specific interface for this purpose, or collected directly from the vehicle's display controller, for example through a serial interface. The second information sent in the periodic announcements is the variation of the speed of the vehicle, collected from an accelerometer present in the module. With this information, the message is mounted as a String variable with 16 bits and sent at a frequency of $51.61 \mathrm{~Hz}$.

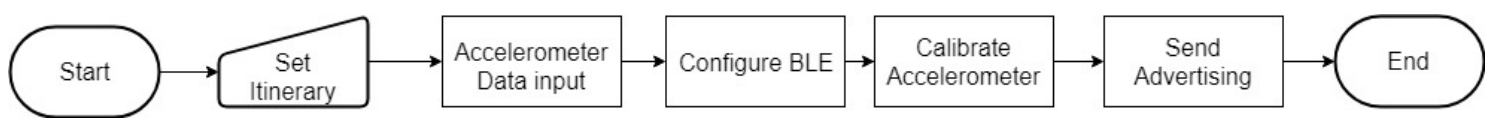

Figure 4. Flowchart of BLE beacons generation in the BlindMobi Box

\subsection{BlindMobi App description}

With the function of reading the beacons sent by the subsystem described in the previous step and designed to be deployed on a smartphone, supported by a mobile operating system (Android or IOS), the proposed application operation (BlindMobi App) can be divided into two stages:

- Initial stage: Once the application is started and the desired transport route information is received by the user, converted from voice to text with the help of a specific API, the application activates BLE communication in the standby state 
of requests from other devices. Then, upon receiving the advertising signal, the application confirms the validity of that signal and extracts the beacon data (RSSI + itinerary + acceleration), for the calculation of the distance, to be detailed in section 4.2. In Figure 5 the Initial stage flowchart of the BlindMobi App is displayed.

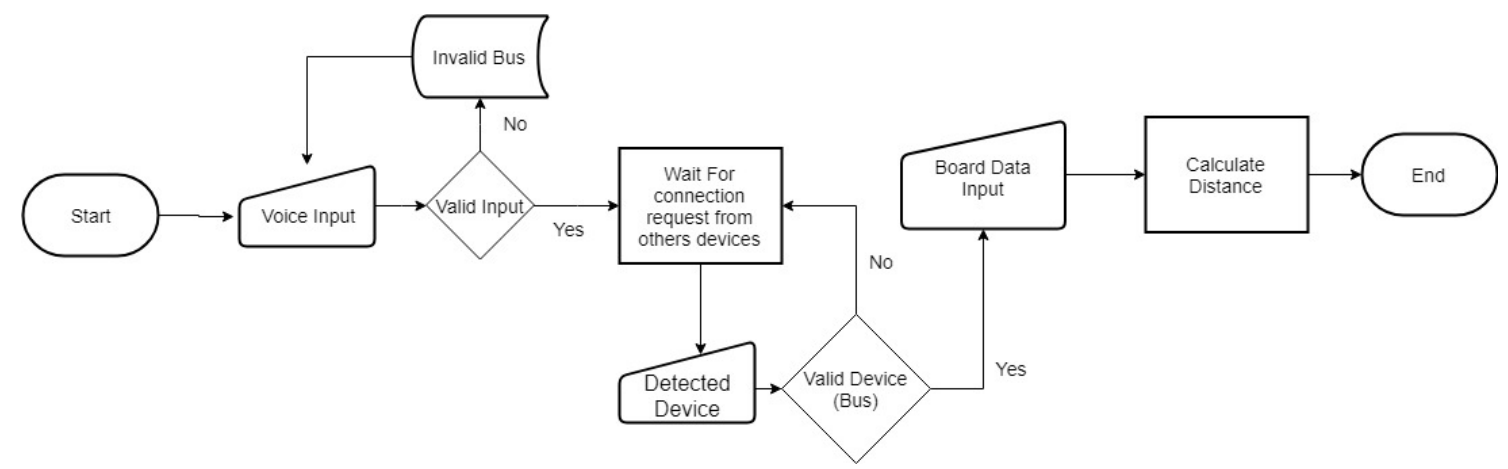

Figure 5. Initial stage flowchart of the BlindMobi App

- Final stage: As described in Figure 6, once the distance is calculated, from the information received by the BLE beacons and the use of machine learning algorithms with fingerprinting technique detailed in the implementation and testing section, are generated the messages "bus is coming" - divulged when the detected bus route coincides with the route of the desired bus, informed by the user in the previous phase and it is at a distance of more than $12 \mathrm{~m}$ and in movement; and the message "bus arrived", if the distance of the bus is less than $12 \mathrm{~m}$ and it is stopped.

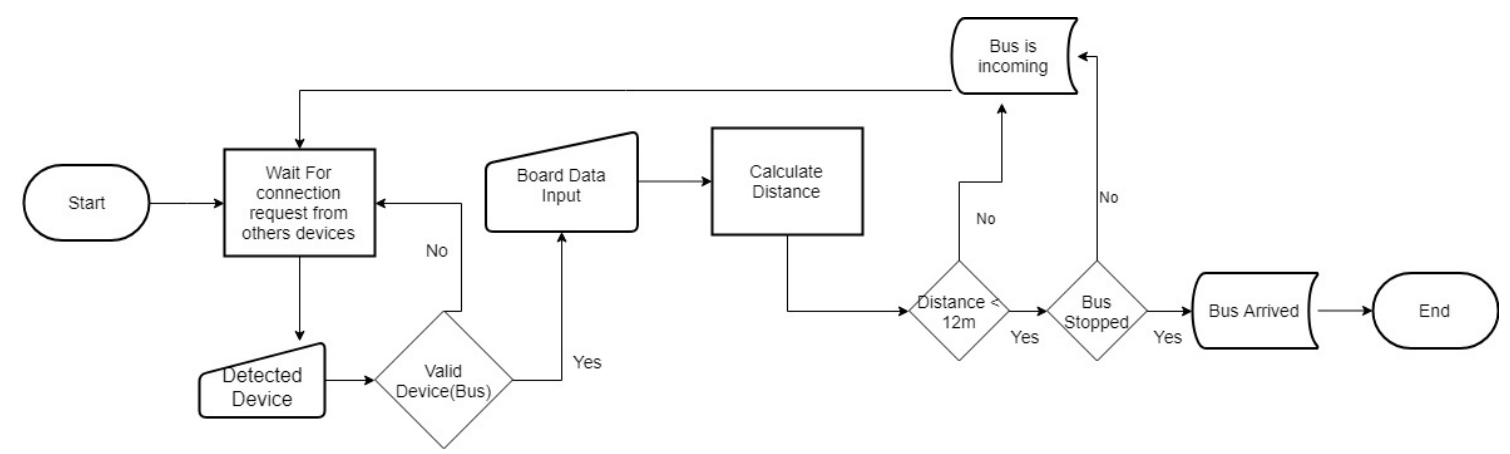

Figure 6. Final stage flowchart of the BlindMobi App

\section{Implementation and Testing}

\subsection{BlindMobi Box Prototype}

To check the functionality of the proposed system, a prototype was built and tested. For this purpose the development kit board PCA 10040, by Nordic, was chosen. The key features of this development kit are:

- nRF52832 flash-based ANT/ANT+, Bluetooth Low Energy SoC solution;

- Buttons, LEDs and I/O interfaces;

- SEGGER J-Link OB debugger with debug out functionality. 
Figure 7 shows the real-size prototype BlindMobi Box hardware, with emphasis on the accelerometer (model MPU6050) and antenna used. Figure 8 shows the location where the BlindMobi Box was installed on the bus used in the field tests, the results of which are described in section 4.3.

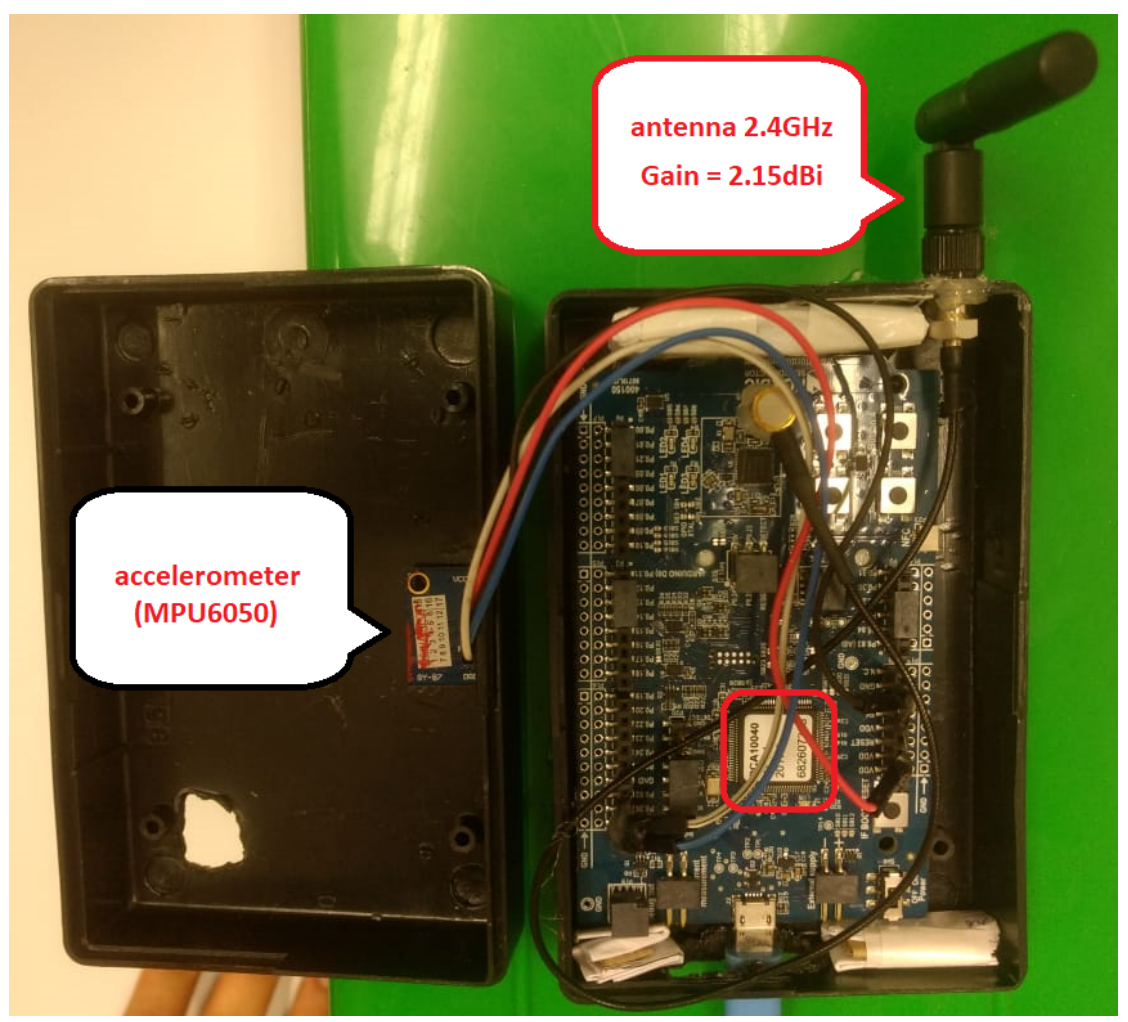

Figure 7. Real-size prototype BlindMobi Box

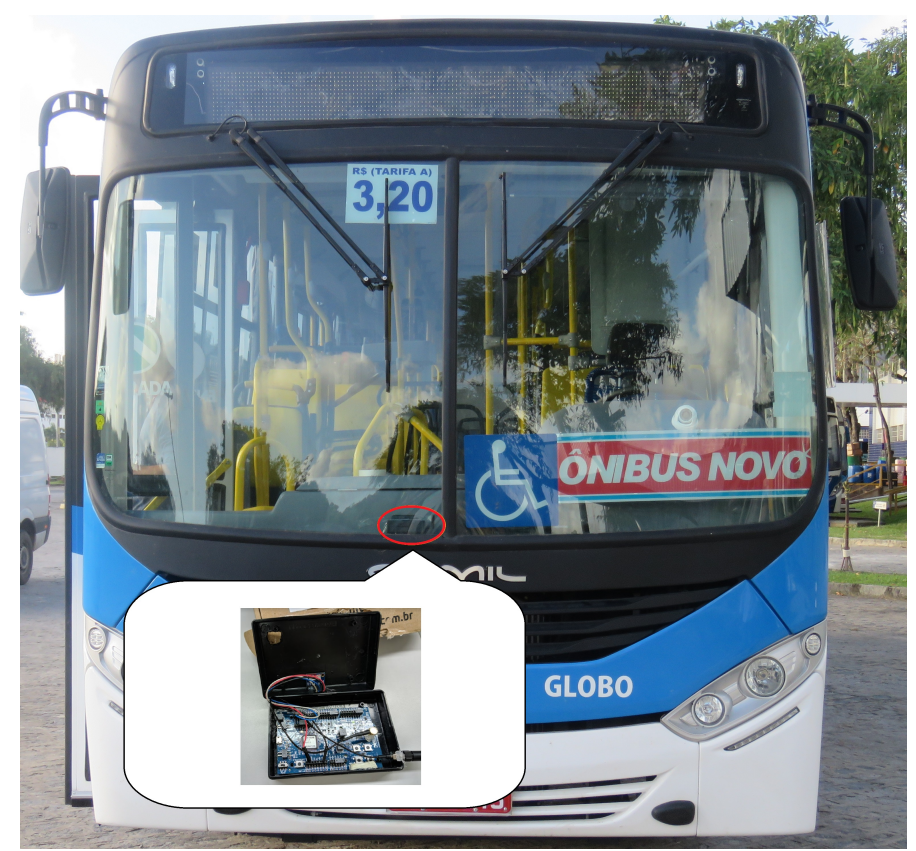

Figure 8. BlindMobi Box installed on the bus 


\subsection{Announcement Messages}

Considering the high degree of variability of the RSSI parameter, due to the frequency band used by the BLE technology that operates in the microwave range $(2.4 \mathrm{GHz})$, being therefore susceptible to propagation degradation as a function of interference, diffraction and reflection and other variations in the environment, machine learning techniques were used to better model the user's distance from the vehicle, depending on the level of RSSI received. Given the nature of the problem and the hardware limitations, two techniques were chosen within the supervised learning paradigm: K-NN (K-Nearest Neighbor) and Decision-Tree.

A K-NN algorithm is an approach to data classification that estimates how likely a data point is to be a member of one group or the other depending on what group data point are nearest to it are in. To evaluate the grouping between these points, which in the proposed system are equivalent to the RSSI and acceleration values ( $\mathrm{x}, \mathrm{y}$ and $\mathrm{z}$ axes), the Euclidean distance was used. Thus, from the values received, five distance classes were assigned: $5 \mathrm{~m}, 10 \mathrm{~m}, 20 \mathrm{~m}, 30 \mathrm{~m}$ and $40 \mathrm{~m}$ - considering the database previously raised in an open field, i.e. the fingerprints - see figure 9 . Then classes $5 \mathrm{~m}$ and $10 \mathrm{~m}$ were associated with the "bus arrived" message while the $20 \mathrm{~m}, 30 \mathrm{~m}$ and $40 \mathrm{~m}$ classes associated with the "bus is coming" message.

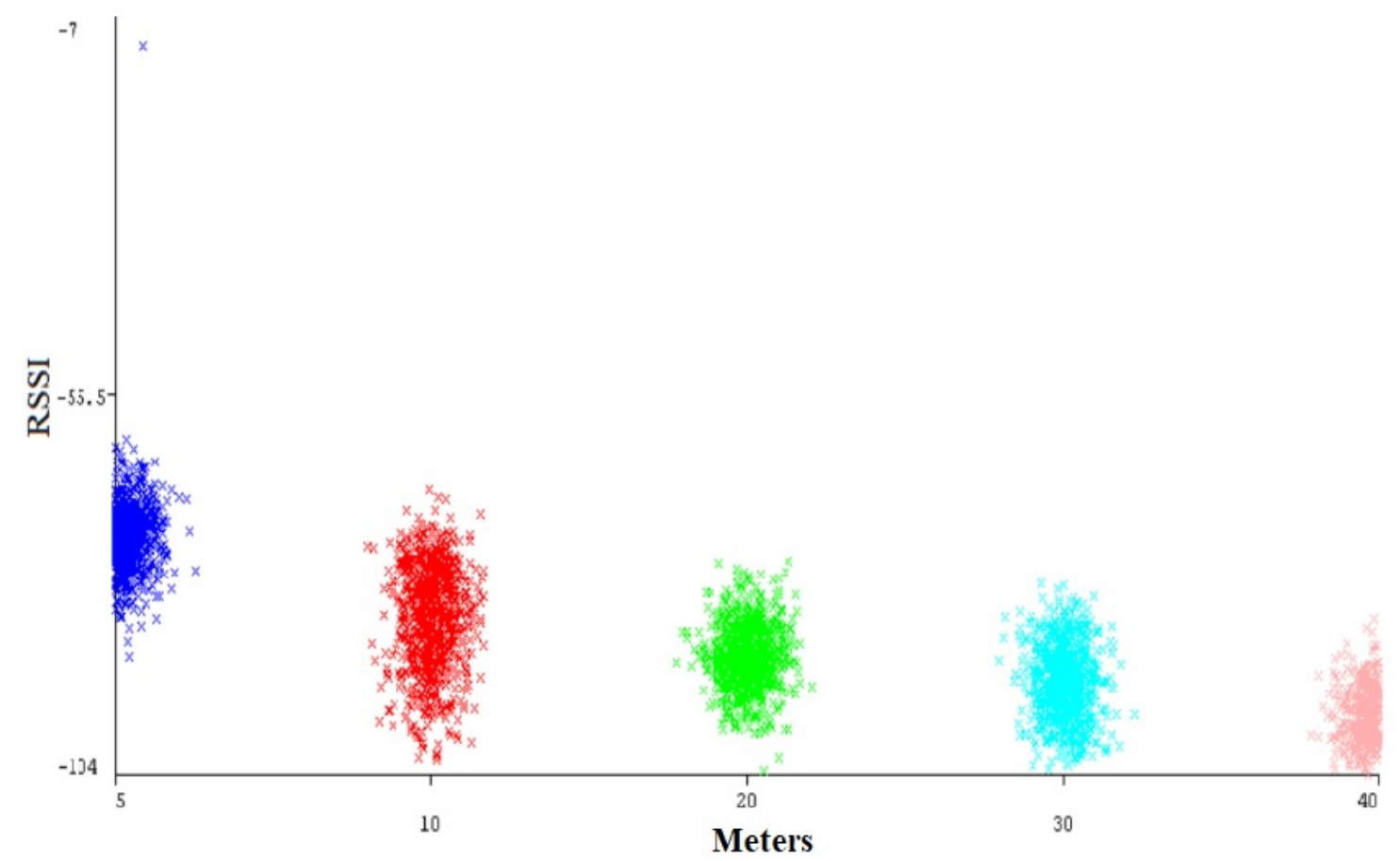

Figure 9. Distance classification according to RSSI values

As a second technique to classify the distance from the RSSI and acceleration values, the C4.5 algorithm of the decision-tree [Xiaoliang et al. 2009] algorithm was chosen. Such choice was due to the simplicity of this technique, where the classification is made from data structures divided into a set of nodes, distributed hierarchically as a function of entropy, previously calculated from the training data. 
In Figure 10, the decision-tree generated from the training data obtained from the prototype detailed in the previous section is presented. In this representation, the nodes in brown were associated to class "bus arrived", whereas the nodes in blue were classified in the class "bus is coming". The higher the degree of certainty of the classification, depending on the values of the training data set, but the darker the color of the node.

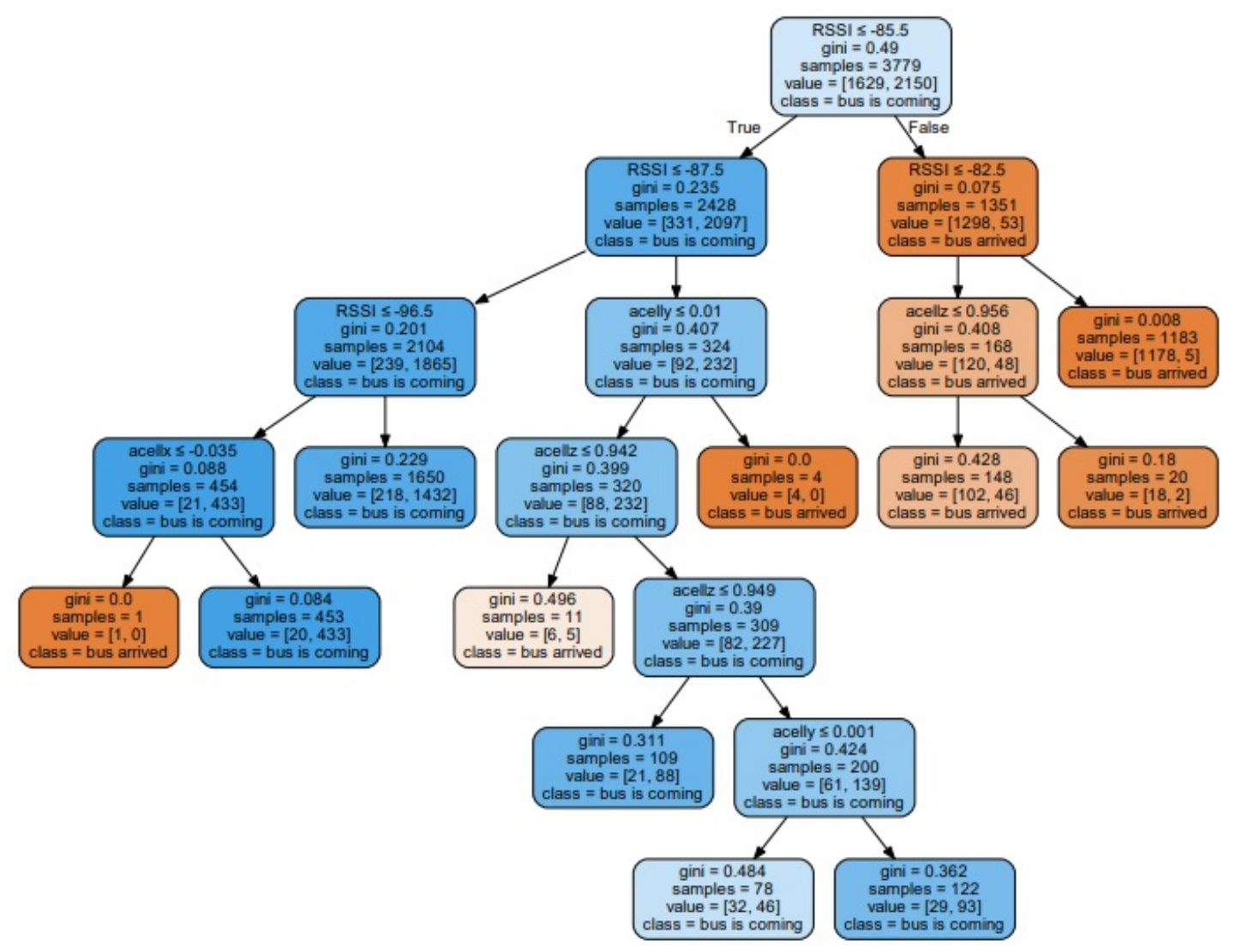

Figure 10. Decision-tree generated from the date-set collected in the field tests 
Finally, from the flowcharts described in section 3.3 and using the classification algorithms described in the previous section, the trial version of BlindMobi App software was developed (See Figure 11, initially supported by the Android 4.3 operating system (or higher). Also, a Google API was used for the translation of text message messages into a voice message ("bus is coming" and "bus arrived").

\subsection{Location Performance Evaluation}

Also known as Scene Analysis, which refers to the type of algorithms that collect the signal features (fingerprints) of a scene and then estimate the target location by matching the real-time signal features got online with the prior collected data set and choose the closest one as result, fingerprinting was the scheme applied for the vehicle location in this research. For validation of the proposed system, a real scenario was used to collect the data set, with the BlindMobi Box device implanted in a bus used in the public transport of passengers of the city of Recife, where real cases of the use of the system were simulated in a private area isolated for this purpose.

To evaluate the performance of each of the algorithms used (KNN and C4.5) we used the data mining program WEKA [Hall et al. 2009], with support for the chosen algorithms. For the KNN technique, the Euclidean distance was used as the selection criterion, with the $\mathrm{K}$ value varied between 1, 3, 5 and 7. In the algorithm of decision tree (C4.5) the criterion of division by entropy was used as criterion of selection. In both techniques, $66 \%$ of the base supplied by the fingerprinting technique was used as the training base, so that the remaining $34 \%$ served as the test base. In order to define the efficiency of each one, the average rating forecast was used.

Firstly, the classification of the distance to each meter in each of the algorithms was evaluated, and the average hit rate was verified, then the distances were converted to only two classes, with values between $5 \mathrm{~m}$ and $10 \mathrm{~m}$ equivalent to the class "bus arrived "and the values between $20 \mathrm{~m}$ and $40 \mathrm{~m}$ equivalent to the "bus is coming" class. The definition of these classes considered the maximum length of a standard bus, used in the collective transport of Recife, which is $9.6 \mathrm{~m}$.

In table 1 the average rating forecast summary is presented, considering the classes "bus is coming", whose objective is to signal to the user the approximation of the vehicle, so that it signals and requests the bus stop, and "bus arrived", so that the same realizes the shipment, of safe form. It is possible to verify that the algorithm C4.5 presented a success rate of $91.5 \%$.

Table 1. Testing the database adapted in different classifiers.

\begin{tabular}{|c|c|}
\hline Classifier & Average rating forecast \\
\hline KNN with $\mathrm{K}=1$ & $83,3 \%$ \\
\hline KNN with $\mathrm{K}=3$ & $86,6 \%$ \\
\hline KNN with $\mathrm{K}=5$ & $89,0 \%$ \\
\hline KNN with $\mathrm{K}=7$ & $89,4 \%$ \\
\hline C4.5 & $91,5 \%$ \\
\hline
\end{tabular}




\section{Conclusions and Future Works}

From the results presented in the field tests performed with the prototype of the proposed system, where a success rate of $91.5 \%$ was obtained in the identification of proximity and stopping of the bus at the point of shipment, the viability of the BlindMobi System was proven. Thus, such a system is very promising for the guarantee of the right of free movement of people with visual impairment, since in addition to a low cost of implantation in the urban environment, as it does not need infrastructure adaptations in bus stops, it is also enough accessible to users, since it does not demand the need of internet connection for its operation, since it is based on direct and connectionless communication, based on Bluetooth Low Energy technology.

As a proposal of future work, it is indicated the evaluation of other machine learning techniques, to improve the classification of approach distance according to RSSI, as well as the development of new features in BlindMobi App software.

\section{Acknowledgments}

This research was supported by the PIBEX Program, developed by PROEXT IFPE, and supported by LIVE (Laboratory of Vehicle Innovations), hosted at UFPE.

\section{References}

Alawi, R. A. (2011). RSSI based location estimation in wireless sensors networks. In 2011 17th IEEE International Conference on Networks. IEEE.

Atzori, L., Iera, A., and Morabito, G. (2010). The internet of things: A survey. Computer Networks, 54(15):2787-2805.

Bertuletti, S., Cereatti, A., Della, U., Caldara, M., and Galizzi, M. (2016). Indoor distance estimated from bluetooth low energy signal strength: Comparison of regression models. In 2016 IEEE Sensors Applications Symposium (SAS). IEEE.

Gill, J. (2003). Requirements for the interconnection of assistive technology devices and information and communication technology systems. http://www.tiresias.org/reports/inter.htm.

Hall, M., Frank, E., Holmes, G., Pfahringer, B., Reutemann, P., and Witten, I. H. (2009). The WEKA data mining software. ACM SIGKDD Explorations Newsletter, 11(1):10.

Jeon, K. E., She, J., Soonsawad, P., and Ng, P. C. (2018). BLE beacons for internet of things applications: Survey, challenges, and opportunities. IEEE Internet of Things Journal, 5(2):811-828.

Kalbani, J. A., Suwailam, R. B., Yafai, A. A., Abri, D. A., and Awadalla, M. (2015). Bus detection system for blind people using RFID. In 2015 IEEE 8th GCC Conference \& Exhibition. IEEE.

Lim, J. T. F., Leong, G. H., and Kiong, T. K. (2008). Accessible bus system: A bluetooth application. In Assistive Technology for Visually Impaired and Blind People, pages 363-384. Springer London.

Pascolini, D. and Mariotti, S. P. (2012). Global estimates of visual impairment: 2010. British Journal of Ophthalmology, 96(5):614-618. 
United Nation, G. (1948). Universal declaration of human rights. Art. 13, [accessed 09 December 2018].

Xiaoliang, Z., Hongcan, Y., Jian, W., and Shangzhuo, W. (2009). Research and application of the improved algorithm c4.5 on decision tree. In 2009 International Conference on Test and Measurement. IEEE.

Zhang, L., Liu, X., Song, J., Gurrin, C., and Zhu, Z. (2013). A comprehensive study of bluetooth fingerprinting-based algorithms for localization. In 2013 27th International Conference on Advanced Information Networking and Applications Workshops. IEEE. 